\title{
Effects of elastic foundation on the snap-through buckling of a shallow arch under a moving point load
}

\author{
Jen-San Chen *, Yuon-Tai Li \\ Department of Mechanical Engineering, National Taiwan University, No. 1, Roosevelt Road, Sec 4, Taipei 10617, Taiwan, ROC
}

Received 26 December 2004; received in revised form 21 April 2005

Available online 1 July 2005

\begin{abstract}
In this paper we study the effects of elastic foundation on the static and dynamic snap-through of a shallow arch under a point load traveling at a constant speed. The deformation of the arch is expressed in a Fourier series. For static analysis when the moving speed of the point load is almost zero, the first four modes in the expansion are sufficient in predicting the equilibrium positions and the critical loads. Unlike the case without elastic foundation, static snapthrough can occur even when the arch is in another stable $\left(P_{1}^{-}\right)$position before the point load moves onto the arch. In the dynamic case when the moving speed of the point load is significant, the numerical simulation of the response does not converge well, especially long after the point load leaves the arch. However, the total energy of the arch converges quite well when only the first eight modes are used in the Fourier series. This observation allows us to establish a sufficient condition against dynamic snap though, although we are unable to predict precisely, with finite number of modes in the series, at what time it will occur when this sufficient condition is not fulfilled.
\end{abstract}

(C) 2005 Elsevier Ltd. All rights reserved.

Keywords: Elastic foundation; Shallow arch; Snap through

\section{Introduction}

An arch subjected to lateral loads may become elastically unstable. If the initial height of the arch is of the same order as the span of the arch, the buckling deformation is nearly inextensional. Sometimes, an arch of this nature is called a ring. On the other hand, an arch is termed shallow if the initial height is much smaller than the span. When the lateral load of a shallow arch reaches a critical value the deformed shape may undergo a sudden jump called snap-through buckling. The buckling deformation of a shallow arch will

\footnotetext{
* Corresponding author. Tel.: +886223 661734; fax: +886223631755.

E-mail address: jschen@ccms.ntu.edu.tw (J.-S. Chen).
} 
be extensional rather than inextensional. Depending on how the lateral load is applied, the snap-through buckling of a shallow arch can be divided into two categories, i.e., static buckling and dynamic buckling. In the case of static buckling, the lateral load is applied in a quasi-static manner. The first theoretical prediction on the static critical load was conducted by Timoshenko (1935), in which a pinned sinusoidal arch was subjected to a uniformly distributed load. Timoshenko's pioneering work was followed and extended by many other researchers, including Fung and Kaplan (1952), Gjelsvik and Bonder (1962), Onat and Shu (1962), Franciosi et al. (1964), Schreyer and Masur (1966), Lee and Murphy (1968), Simitses (1973), and Chen and Lin (2005). Experimental results have been reported by Roorda (1965).

In the case when the lateral load is applied dynamically instead of in the quasi-static manner, the critical load will be different from the one predicted statically, see Hoff and Bruce (1954), Humphreys (1966), Lock (1966), Hsu (1967, 1968), Hsu et al. (1968), Huang and Nachbar (1968), Fulton and Barton (1971), Sundararajan and Kumani (1972), Lo and Masur (1976), Johnson and Mclvor (1978), Johnson (1980), Gregory and Plaut (1982), Donaldson and Plaut (1983), Patricio et al. (1998), Xu et al. (2002), Lin and Chen (2003), Chen and Lin (2004a), and Chen and Liao (2005). A comprehensive review on the dynamic instability of shallow arches can be found in the book by Simitses (1990).

In all these previous researches, the lateral loading, either distributed or concentrated, is assumed to be fixed in space. In 1979, Plaut studied the effect of point load position on the stability of a shallow arch. This paper described the behavior of a shallow arch under a point load moving through the span quasi-statically. Chen and Lin (2004b) extended Plaut's work to study the dynamic snap-through buckling of a shallow arch under a point force moving with high speed. They reported that when the point load is greater than a dynamic critical load there exists a finite speed zone within which the arch runs the risk of dynamic snapthrough. The establishment of the sufficient condition against dynamic snap through is based on the concept of comparing the total energy gained by the arch and a critical energy barrier. In this paper we extend Chen and Lin's work (2004b) to discuss the effects of an elastic foundation (Hetenyi, 1946) on the static and dynamic stability of a pinned shallow arch under a moving point force. The consideration of an elastic foundation represents a more realistic modeling of many civil structures. For instance, the cement concrete pavement on a rolling-hill terrain can be modeled as a curved beam or plate on elastic foundation. Due to axial compressive forces, the concrete pavements sometimes experience buckling, which is also called blowup (Kerr and Dallis, 1985). The situation can be aggravated by high speed traveling of heavy vehicles. The detailed study conducted by Simitses (1973) on the stability of a shallow arch resting on an elastic foundation and under a space-fixed distributed load is a typical example in this regard.

\section{Equations of motion}

Fig. 1 shows an elastic shallow arch with the two pinned-ends being separated by a distance $L$. The arch is assumed to rest on a Winkler-type elastic foundation with elastic constant $\beta^{*}$. The initial shape of the unloaded arch is $y_{0}(x)$. The arch is subjected to a point force $Q^{*}$ traveling from $x=0$ to $x=L$ with a constant speed $c^{*}$. The equation of motion of the arch can be written as

$$
\rho A y_{, t t}=-E I\left(y-y_{0}\right)_{, x x x}+p^{*} y_{, x x}-Q^{*} \delta\left(x-c^{*} t\right)-\beta^{*}\left(y-y_{0}\right) .
$$

The parameters $E, \rho, A$, and $I$ are Young's modulus, mass density, area, and moment of inertia of the crosssection of the arch. $\delta$ is the Dirac delta function. In Eq. (1), we assume that the effect of the axial stress wave on the lateral vibration is negligible. Chen and Lin (2004b) argued that for a curved beam with slenderness ratio 10 , the effect of axial stress wave on the lateral vibration is negligible unless the traveling speed of the point force is in the range of ten times of the flexural wave speed. In this paper we assume that the moving speed of the point force is well below this range. Under such condition the axial force $p^{*}$ can be considered as independent of the load position $x$ and can be calculated as 


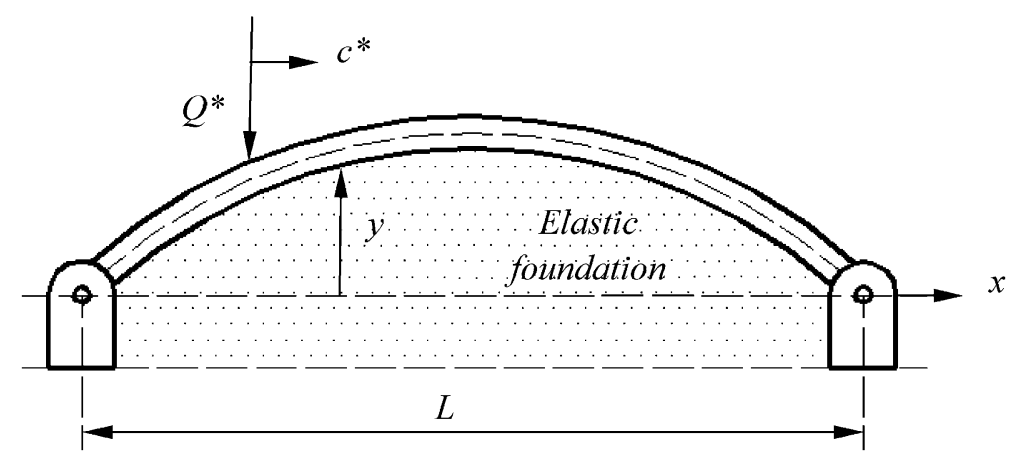

Fig. 1. Schematic diagram of a shallow arch resting on an elastic foundation and under a moving point load.

$$
p^{*}(t)=\frac{A E}{2 L} \int_{0}^{L}\left(y_{, x}^{2}-y_{0, x}^{2}\right) \mathrm{d} x .
$$

The boundary conditions for $y$ at $x=0$ and $L$ are

$$
y(0)-y_{0}(0)=y_{, x x}(0)-y_{0, x x}(0)=y(L)-y_{0}(L)=y_{, x x}(L)-y_{0, x x}(L)=0 .
$$

Eqs. (1) and (2) can be nondimensionalized to the forms

$$
\begin{aligned}
& u_{, \tau \tau}=-\left(u-u_{0}\right)_{, \xi \xi \xi \xi}+p u_{, \xi \xi}-\frac{\pi}{2} Q \delta(\xi-c \tau)-\beta\left(u-u_{0}\right), \\
& p=\frac{1}{2 \pi} \int_{0}^{\pi}\left(u_{, \xi}^{2}-u_{0, \xi}^{2}\right) \mathrm{d} \xi,
\end{aligned}
$$

where

$$
u=\frac{y}{r}, \quad u_{0}=\frac{y_{0}}{r}, \quad \xi=\frac{\pi x}{L}, \quad \tau=\frac{\pi^{2} t}{L^{2}} \sqrt{\frac{E I}{A \rho}}, \quad \beta=\frac{\beta^{*} L^{4}}{\pi^{4} E I}, \quad c=\frac{c^{*} L}{\pi} \sqrt{\frac{A \rho}{E I}}, \quad p=\frac{p^{*} L^{2}}{\pi^{2} E I}, \quad Q=\frac{2 Q^{*} L^{3}}{\pi^{5} E I r},
$$

$r$ is the radius of gyration of the cross-section. $p=1$ corresponds to the Euler buckling load for a perfectly straight simply supported beam. $c=1$ corresponds to the speed of the flexural wave of the curved-beam with wavelength $L$. It is noted that $Q$ is positive when the concentrated load points downward in Fig. 1. The unstressed shape of the arch before the lateral load is applied is assumed to be in the form

$$
u_{0}(\xi)=h \sin \xi,
$$

$h$ is the rise parameter of the arch.

It is assumed that the shape of the loaded arch can be expanded as

$$
u(\xi, \tau)=\lim _{N \rightarrow \infty} \sum_{n=1}^{N} \alpha_{n}(\tau) \sin n \xi .
$$

After substituting Eqs. (7) and (8) into (4) and (5) we obtain the equations governing $\alpha_{n}$,

$$
\ddot{\alpha}_{n}=-n^{4} \alpha_{n}-n^{2} p \alpha_{n}-q_{n}-\beta \alpha_{n}, \quad n=1,2,3, \ldots,
$$


where

$$
\begin{aligned}
& p=\frac{1}{4} \sum_{k=1}^{\infty} k^{2} \alpha_{k}^{2}-\frac{h^{2}}{4}, \\
& q_{1}=Q \sin e-h-\beta h, \\
& q_{n}=Q \sin n e, \quad n=2,3, \ldots \\
& e(\tau)=c \tau .
\end{aligned}
$$

The parameter $0<e(\tau)<\pi$ represents the position of the point load on the arch. The overhead dot in Eq. (9) represents differentiation with respect to $\tau$. The initial conditions for Eq. (9) are

$$
\alpha_{1}(0)=h, \quad \alpha_{n}(0)=0 \text { for } n=2,3, \ldots ; \quad \dot{\alpha}_{n}(0)=0 \text { for } n=1,2,3, \ldots
$$

\section{Equilibrium equations}

We first consider the case when the moving speed $c$ of the point load is small and the acceleration term in Eq. (9) can be neglected. The equilibrium equations governing $\alpha_{n}$ can then be written as

$$
n^{4} \alpha_{n}+n^{2} p \alpha_{n}+q_{n}+\beta \alpha_{n}=0, \quad n=1,2,3, \ldots
$$

In the case when $e=0$, i.e., the arch is free from the point load, there could exist one-mode solutions involving only $\alpha_{1}$, and two-mode solutions involving $\alpha_{1}$ and $\alpha_{j}$, where $j \neq 1$. For a specified $h$, we can define a $\beta_{1}$ such that there are three one-mode solutions $\left(P_{0}, P_{1}^{+}, P_{1}^{-}\right)$as long as $\beta<\beta_{1}$ (Chen and Lin, 2004a), where

$$
\beta_{1}=\left(\frac{h}{4}\right)^{2}-1
$$

On the other hand, there exists only one one-mode solution $P_{0}$ if $\beta>\beta_{1}$. Similarly, we can define a $\beta_{j}$ such that the two-mode solutions $P_{1 j}^{ \pm}$exist if and only if $\beta<\beta_{j}$, where

$$
\frac{\left(j^{2}-1\right)^{2}\left(\beta_{j}-j^{2}\right)^{2}}{\left(1-2 j^{2}\right) \beta_{j}+j^{4}-2 j^{2}}=\frac{j^{2} h^{2}}{4} \text {. }
$$

Chen and Lin (2004a) also reported that there exist at most two stable equilibrium positions $P_{0}$ and $P_{1}^{-}$if $\beta<\beta_{2}$.

In the case when the point load is on the arch Eq. (15) represents an infinite number of coupled nonlinear equations for the infinite number of coordinates $\alpha_{n}$. While it is in general impossible to solve for the infinite number of $\alpha_{n}$ simultaneously, it is possible to use a deduction method to derive the equation for $\alpha_{1}$. It is noted that equilibrium position of a shallow arch under a point force at the mid-point is a classical problem and has been presented in (Fung and Kaplan, 1952). However, a more general case when the point load is at an arbitrary position of the arch is not available in the literature except in Plaut (1979) and Chen and Lin (2004b). The following methodology is a concise extension of the method adopted by Chen and Lin (2004b) to the case with elastic foundation.

$N=1$ : We first assume that the number of modes $N$ used in Eq. (8) is 1 . Then the solution $\alpha_{1}$ can be solved from the following cubic equation:

$$
f_{1}\left(\alpha_{1}\right)=\alpha_{1}+\frac{\alpha_{1}}{4}\left(\alpha_{1}^{2}-h^{2}\right)+q_{1}+\beta \alpha_{1}=0 .
$$

There are at most three one-mode equilibrium positions for $N=1$. 
$N=2$ : For the case when $N=2$ there are at most five equilibrium positions. After eliminating the axial thrust $p$ in Eq. (15) for $n=1$ and 2, we can derive the relation between $\alpha_{1}$ and $\alpha_{2}$ as

$$
\alpha_{2}=-\frac{\alpha_{1} q_{2}}{\left[3(4-\beta) \alpha_{1}-4 q_{1}\right]} .
$$

After substituting Eq. (19) into the first equation in (15) we obtain the equation for $\alpha_{1}$ as

$$
f_{2}\left(\alpha_{1}\right)=\left[3(4-\beta) \alpha_{1}-4 q_{1}\right]^{2} f_{1}\left(\alpha_{1}\right)+q_{2}^{2} \alpha_{1}^{3}=0 .
$$

$N=k$ : After eliminating $p$ in Eq. (15) for $n=1$ and $j$ we can derive the relation between $\alpha_{1}$ and $\alpha_{j}$ as

$$
\alpha_{j}=\frac{-\alpha_{1} q_{j}}{\left[\left(j^{2}-1\right)\left(j^{2}-\beta\right) \alpha_{1}-j^{2} q_{1}\right]}, \quad j=2,3, \ldots, k
$$

After substituting Eq. (21) into the first equation in (15) we obtain the equation for $\alpha_{1}$ as

$$
f_{k}\left(\alpha_{1}\right)=\left[\left(k^{2}-1\right)\left(k^{2}-\beta\right) \alpha_{1}-k^{2} q_{1}\right]^{2} f_{k-1}\left(\alpha_{1}\right)+\frac{k^{2} q_{k}^{2} \alpha_{1}^{3}}{4} \prod_{j=2}^{k-1}\left[\left(j^{2}-1\right)\left(j^{2}-\beta\right) \alpha_{1}-j^{2} q_{1}\right]^{2}=0,
$$

$f_{k-1}\left(\alpha_{1}\right)=0$ is the equation for $\alpha_{1}$ when $N=k-1$. After successive substitution Eq. (22) can also be rewritten as

$$
f_{k}\left(\alpha_{1}\right)=f_{1}\left(\alpha_{1}\right) \prod_{j=2}^{k}\left[\left(j^{2}-1\right)\left(j^{2}-\beta\right) \alpha_{1}-j^{2} q_{1}\right]^{2}+\sum_{i=2}^{k}\left\{\frac{i^{2} q_{i}^{2} \alpha_{1}^{3}}{4} \prod_{\substack{j=2 \\ j \neq i}}^{k}\left[\left(j^{2}-1\right)\left(j^{2}-\beta\right) \alpha_{1}-j^{2} q_{1}\right]^{2}\right\}=0 .
$$

Eq. (23) is a polynomial of order $2 k+1$, admitting at most $2 k+1$ real $\alpha_{1}$. For those real $\alpha_{1}$, the corresponding $\alpha_{j}$ are also real when the arch is loaded as can be calculated from Eq. (21). On the other hand for an unloaded arch, both $q_{j}$ in the numerator and the bracket term in the denominator of Eq. (21) are zero. Therefore, Eq. (21) is not applicable for a free arch. For a free arch a real $\alpha_{1}$ may correspond to a purely imaginary $\alpha_{j}$. Obviously, only those solutions with real $\alpha_{j}$ are real equilibrium configurations.

\section{Effects of $\beta$ on equilibrium positions of the loaded arch}

The first question in numerical calculation is how many modes should be included in the expansion (8). Chen and Lin (2004b) reported that in the case without elastic foundation the first two modes are sufficient in predicting the stable equilibrium positions and the static buckling load. This is no longer true when the elastic foundation $\beta$ is included in the model, as explained in the following.

The thick lines in Fig. 2 are the $\alpha_{1}$ 's as functions of load position $e$ for the case when $h=8$ and $\beta=0.5$. The special $\beta_{j}$ 's as defined in Eqs. (16) and (17) are $\beta_{1}=3, \beta_{2}=0.96, \beta_{3}=2.65$. All other $\beta_{j}$ 's are negative. Fig. 2(a), (b), and (c) are for $Q=18,18.16$, and 20, respectively. The number of modes $N$ used in the expansion is 4 . While there are at most nine equilibrium positions for $N=4$, only seven of them are real. The equilibrium positions with the same loading condition but with $\beta=0$ have been presented by Chen and Lin (2004b) and are reproduced in Fig. 2 with thin lines. By comparing the thick lines and the thin lines, we can observe some effects of the elastic foundation on the equilibrium positions. For the unloaded arch there are three one-mode solutions $\left(P_{0}, P_{1}^{+}, P_{1}^{-}\right)$and two pairs of two-mode solutions $\left(P_{12}^{ \pm}, P_{13}^{ \pm}\right)$, among them only $P_{0}$ and $P_{1}^{-}$are stable while all others are unstable. As the point load moves across the arch, these onemode and two-mode solutions will involve all the harmonic modes in expansion (8). However, we retain the names of the equilibrium positions when the point load moves across the arch because these components 

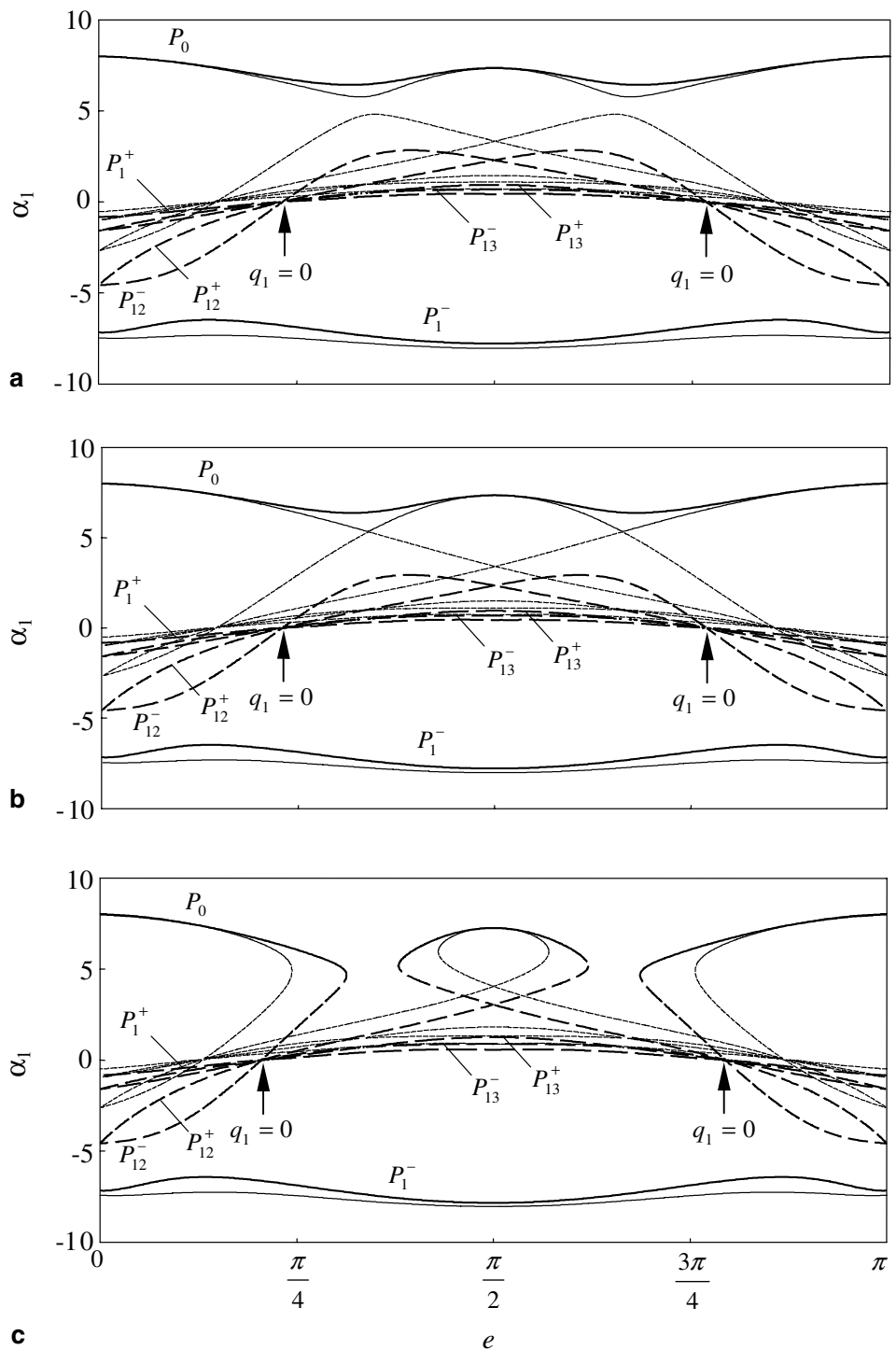

Fig. 2. $\alpha_{1}(e)$ for an arch with $h=8$ and $\beta=0.5$. (a) $Q=18$. (b) $Q=18.16$. (c) $Q=20$.

$\left(\alpha_{1}\right.$ for originally one-mode-solutions and $\left(\alpha_{1}, \alpha_{j}\right)$ for the originally two-mode solutions) remain dominant compared to others. We use solid and dashed lines to denote stable and unstable solutions.

For the case with $\beta=0$ and the arch is in $P_{0}$ position before the point load moves onto the arch, the static critical load is 18.16. This is defined as the point load at which trans-critical bifurcation between the stable $P_{0}$ solution and the unstable $P_{12}^{-}$solution occurs, as shown by the thin lines in Fig. 2(b). Therefore, for a point load smaller than 18.16, say, 18 as in Fig. 2(a), no bifurcation will occur as the point load moves across the arch. This is still the case when elastic foundation $\beta=0.5$ is present, as shown in Fig. 2(a), although the deflection of $\alpha_{1}$ is slightly smaller due to the additional support from the elastic foundation. When the point load is increased to 18.16 as in Fig. 2(b), the load is no longer large enough for the transcritical bifurcation between $P_{0}$ and $P_{12}^{-}$to occur when $\beta=0.5$. Further calculation shows that the new static 
critical load for $\beta=0.5$ is 19.77 . In other words, as one might expect, the elastic foundation tends to prevent the shallow arch from snapping from position $P_{0}$ to $P_{1}^{-}$. This critical load is denoted by $Q_{\mathrm{cr}}^{\left(P_{0}\right)}$, emphasizing that the arch is in $P_{0}$ position before the point load moves onto the arch. For an even larger point load, say $Q=20$, Fig. 2(c) shows that snap through from $P_{0}$ to $P_{1}^{-}$occurs at load position $e=0.99$. In the case $\beta=0$ this saddle-node bifurcation occurs earlier at load position $e=0.76$. It is noted that at the load positions $e$ which render $q_{1}=0$ (Eq. (11)), all but two $\alpha_{1}$-curves intersect at the point $\alpha_{1}=0$. This phenomenon can be proved mathematically to be true as in the case when $\beta=0$ (Chen and Lin, 2004b).

It is noted that if $P_{1}^{-}$is the initial position of the arch, then no snap through will occur when the point load moves along the arch quasi-statically. This is true for $\beta=0$ and 0.5 . A more interesting phenomenon is
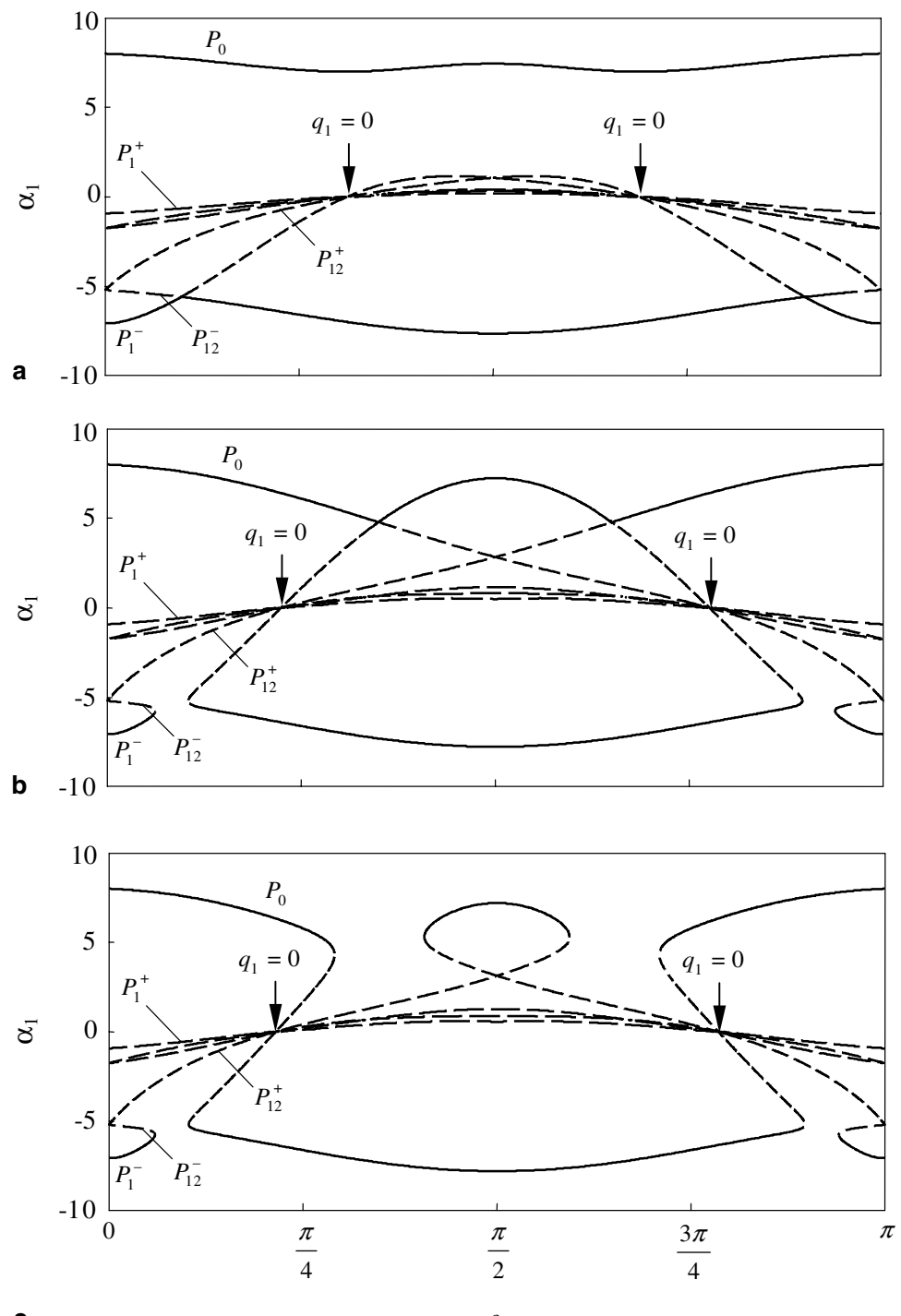

C

$e$

Fig. 3. $\alpha_{1}(e)$ for an arch with $h=8$ and $\beta=0.64$. (a) $Q=Q_{\mathrm{cr}}^{\left(P^{-}\right)}=15.81$. (b) $Q=Q_{\mathrm{cr}}^{\left(P_{0}\right)}=20.26$. (c) $Q=21$. 
that the stable position $P_{1}^{-}$tends to approach the unstable position $P_{12}^{-}$as the point load moves to the right. This trend is more obvious when $\beta$ increases, as will be shown in Fig. 3.

To examine the accuracy of using the first four modes in expansion (8), we redo the calculation by using eight modes in the expansion. It is found that the $\alpha_{1}$ presented in Fig. 2 are almost unchanged. Therefore, in the following calculations, only the first four modes are used. It is noted that Chen and Lin (2004b) reported that the first two modes in the expansion are ultimately important and all the physical essences are retained even when only the first two modes are used in the expansion. This is however not the case when elastic foundation is present, especially when $\beta$ is large. Our experience shows that at least four modes should be retained in the expansion in order to obtain satisfactory accuracy in quasi-static analysis, at least to the range of $\beta$ of our interest.
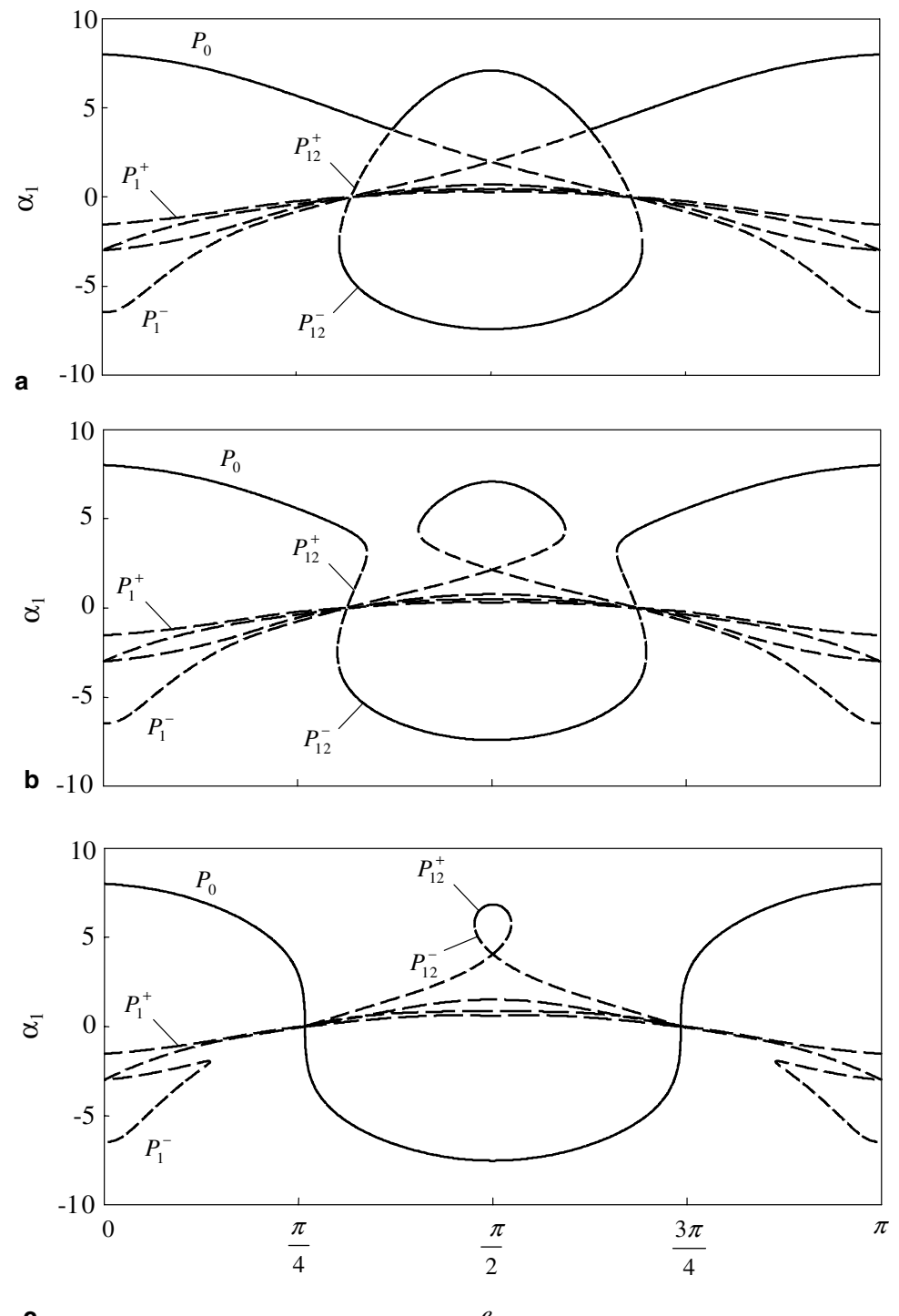

$e$

Fig. 4. $\alpha_{1}(e)$ for an arch with $h=8$ and $\beta=1.5$. (a) $Q=Q_{\mathrm{cr}}^{\left(P_{0}\right)}=23.7$. (b) $Q=24$. (c) $Q=Q_{\mathrm{inf}}=27.59$. 
In Fig. 3, we show the equilibrium positions for the case with $\beta=0.64$. Fig. 3(a) shows the case when $Q=15.81$. At this load, solution $P_{1}^{-}$merges with $P_{12}^{-}$, while no bifurcation occurs along the $P_{0}$ curve. This load may be considered as critical in the sense that for a load larger than 15.81 and the initial position of the arch is $P_{1}^{-}$, then the arch may snap from $P_{\left(p_{-}^{-}\right.}^{-}$to $P_{0}$ when the point load moves across the arch quasi-statically. We may denote this critical load as $Q_{\mathrm{cr}}^{\left(P_{1}^{-}\right)}$. It is noted that the merging of $P_{1}^{-}$and $P_{12}^{-}$will never occur in the case when $\beta=0$ and 0.5 (Fig. 2). Further increase of the point load to 20.26, as in Fig. 3(b), the merging

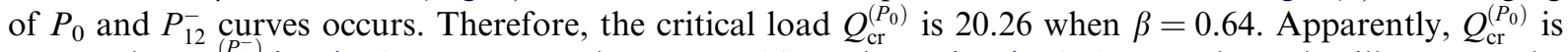
greater than $Q_{\mathrm{cr}}^{\left(P^{-}\right)}$in Fig. 3. For an even larger $Q=21$, as shown in Fig. 3(c), snap through will occur as the point load moves across the arch no matter the initial position of the arch is $P_{0}$ or $P_{1}^{-}$.

Fig. 4 shows the equilibrium positions for $\beta=1.5$, which is greater than $\beta_{2}$ and smaller than $\beta_{1}$ and $\beta_{3}$. Therefore there are three one-mode solutions $\left(P_{0}, P_{1}^{+}, P_{1}^{-}\right)$and one pair of two-mode solutions $\left(P_{13}^{ \pm}\right)$for the unloaded arch, among them only $P_{0}$ is stable. It is noted that the $\alpha_{1}$ for the nonreal $P_{12}^{ \pm}$solutions are complex conjugate pairs before the load moves onto the arch. Fig. 4(a) shows the situation when $Q=23.7$. As this load reaches $e=0.96$, the complex conjugate pair $\alpha_{1}$ of solutions $P_{12}^{ \pm}$become real, among them $P_{12}^{+}$is unstable and $P_{12}^{-}$is stable. As the load moves further to the right at $e=1.17$, trans-critical bifurcation between stable $P_{0}$ and unstable $P_{12}^{+}$occurs. This load becomes the static critical load $Q_{\mathrm{cr}}^{\left(P_{0}\right)}$ for this $\beta$. The behavior of solutions $\alpha_{1}\left(P_{0}\right)$ and $\alpha_{1}\left(P_{12}^{ \pm}\right)$can be examined by a root loci in the complex plane with load position $e$ as a control parameter, as shown in Fig. 5. The symbols $\times$ represent the solutions for $e=0$ and the open circles signify the situation when merging of root loci occurs. It is noted that while $\alpha_{1}\left(P_{12}^{ \pm}\right)$at $e=0$ are real, the corresponding $\alpha_{2}$ are purely imaginary. Therefore, $P_{12}^{ \pm}$are not real for the unloaded arch.

Fig. 4(b) shows the equilibrium positions for a larger load $Q=24$, which breaks the trans-critical bifurcation into a pair of saddle-node bifurcation. The position corresponding to the left saddle node bifurcation is $e=1.06$. It is noted that there are another pair of saddle-node bifurcation points in the lower branch at $e=0.95$ and 2.19. Therefore, when the point load moves across the arch quasi-statically, the arch will jump from upper position to lower position at $e=1.06$, and then jump back to the upper position at $e=2.19$. As $Q$ increases further to 27.59, as shown in Fig. 4(c), the upper and lower bifurcation points approach each other and the solution curve exhibits an inflection point with vertical tangent. We denote this special $Q$ as $Q_{\text {inf. }}$. Although there is no jump phenomenon at $Q_{\text {inf }}$, the arch still experiences dramatic deformation from

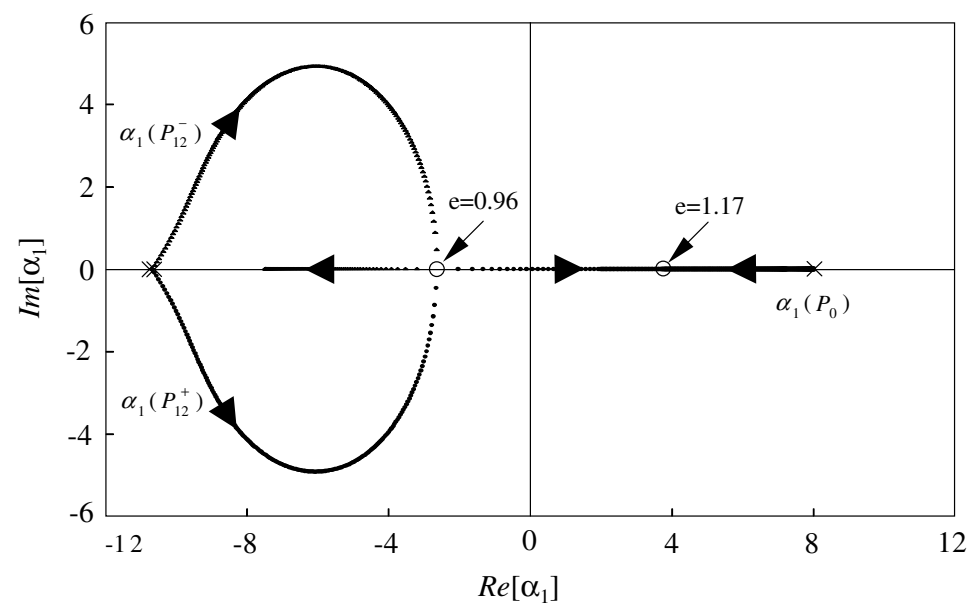

Fig. 5. Root loci for solutions $\alpha_{1}\left(P_{0}\right)$ and $\alpha_{1}\left(P_{12}^{ \pm}\right)$with $e$ as the control parameter. Physical parameters are $h=8, \beta=1.5, Q=23.7$, corresponding to Fig. 4(a). 

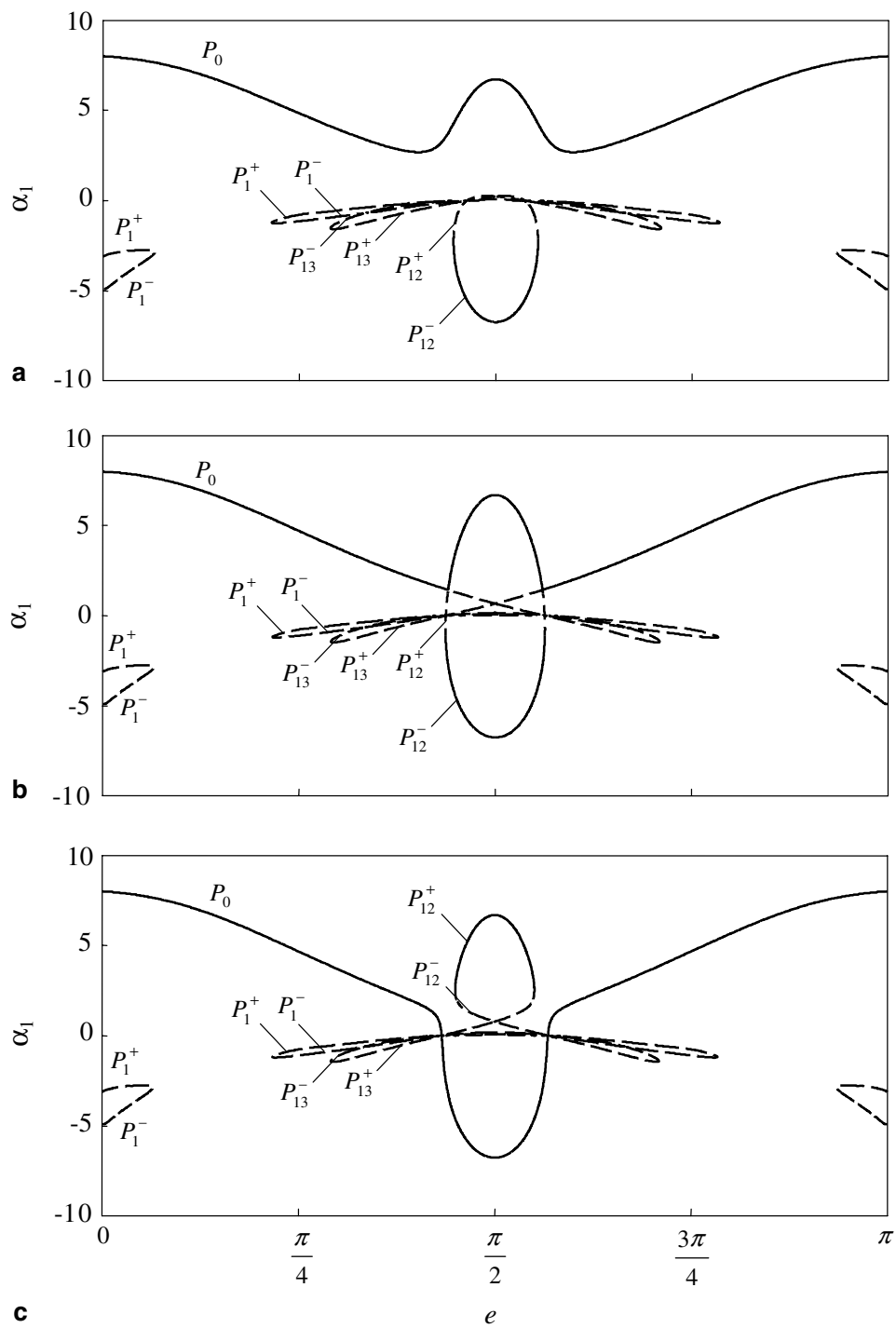

Fig. 6. $\alpha_{1}(e)$ for an arch with $h=8$ and $\beta=2.8$. (a) $Q=30.6$. (b) $Q=Q_{\mathrm{cr}}^{\left(P_{0}\right)}=31$. (c) $Q=31.1$.

positive to negative $\alpha_{1}$. Further increase of point load results in slightly less dramatic deformation with the slope at the inflection point nonvertical.

In Fig. 6, we increase $\beta$ to 2.8, which is greater than $\beta_{2}$ and $\beta_{3}$, but smaller than $\beta_{1}$. Therefore, there are three one-mode solutions $\left(P_{0}, P_{1}^{+}, P_{1}^{-}\right)$but no real two-mode solutions for the unloaded arch. There still exists a $Q_{\mathrm{cr}}^{\left(P_{0}\right)}$ equal to 31. Fig. 6(a), (b), and (c) show the response curves for $Q$ smaller, equal to, and larger than $Q_{\mathrm{cr}}^{\left(P_{0}\right)}$. As load $Q=30.6$ moves across the arch, as shown in Fig. 6(a), the unstable $P_{1}^{+}$and $P_{1}^{-}$merge at $e=0.29$ and become a complex conjugate pair thereafter. These two solutions become real again when the load reaches $e=0.65$. As the load moves further to the right, the originally complex solutions of $P_{12}^{ \pm}$ and $P_{13}^{ \pm}$become real at $e=1.41$, and 0.91, respectively. As $Q=Q_{\mathrm{cr}}^{\left(P_{0}\right)}$, as shown in Fig. 6(b), trans-critical 
bifurcation between solutions $P_{0}$ and $P_{12}^{+}$occurs. The $Q_{\text {inf }}$ for this $\beta$ is 31.04. Snap-through buckling occurs only in the small range of $Q$ between $Q_{\mathrm{cr}}^{\left(P_{0}\right)}$ and $Q_{\text {inf. }}$.

In Fig. 7, we increase $\beta$ further to 5. For this $\beta$ only the one-mode solution $P_{0}$ is real when the arch is not loaded. For a smaller $Q=43$, the $P_{0}$ curve will form a cusp at the midpoint, as shown in Fig. 7(a). We denote this $Q$ as $Q_{\text {cusp }}^{(\mathrm{u})}$, which means that the cusp is in the upper position. Further increase of $Q$ to 47.5, the $P_{0}$ curve will form a loop around the mid-point while another separate loop (unstable) forms in the lower branch, as shown in Fig. 7(b). Jump phenomenon will occur at $e=1.61$ when the point force moves quasi-statically. Further increase of $Q$ to 49 , the loop in the original $P_{0}$ curve will separate, and the lower loop will connect to the $P_{0}$ curve, as shown in Fig. 7(c). As a consequence, the arch will jump back-
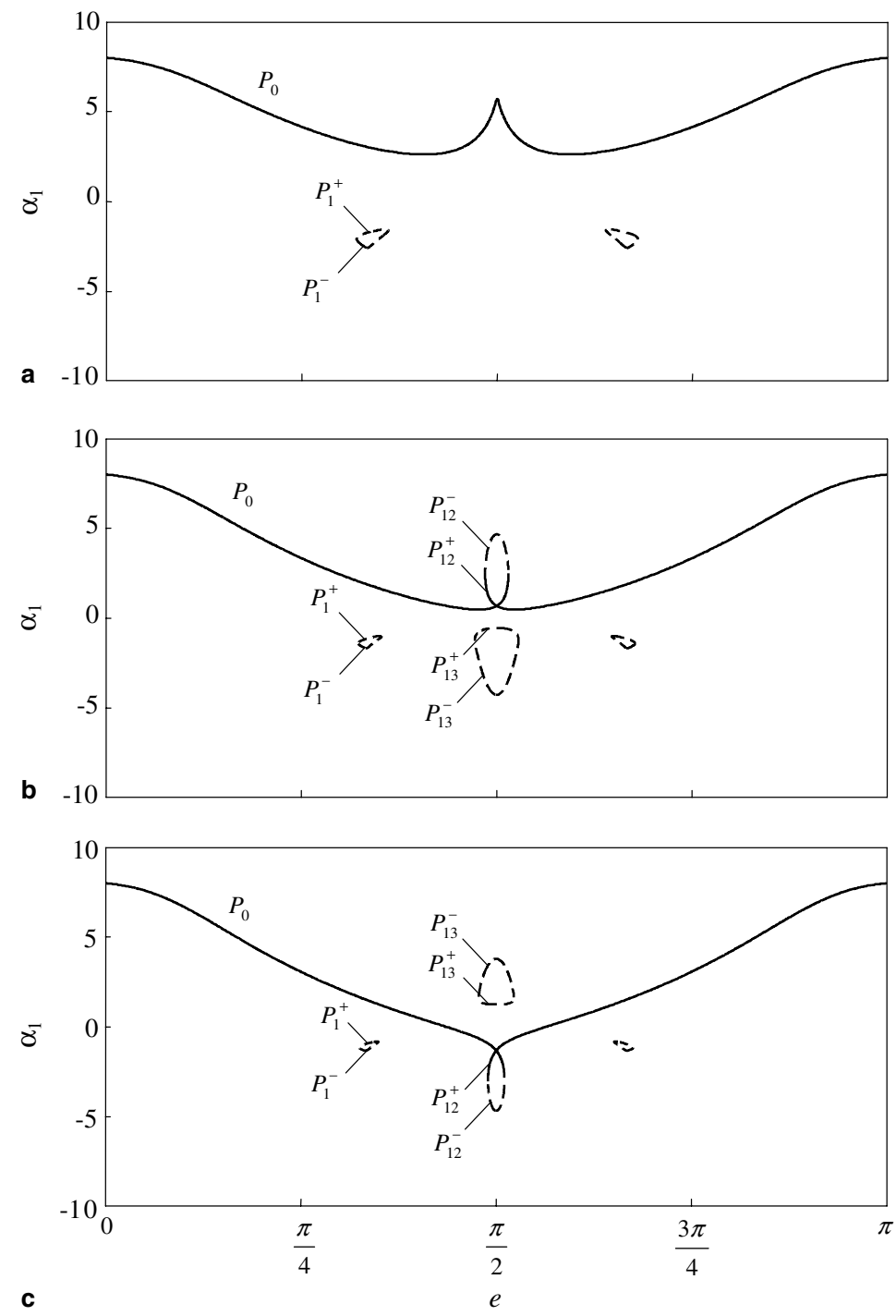

Fig. 7. $\alpha_{1}(e)$ for an arch with $h=8$ and $\beta=5$. (a) $Q=Q_{\text {cusp }}^{(\text {u) }}=43$. (b) $Q=47.5$. (c) $Q=49$. 


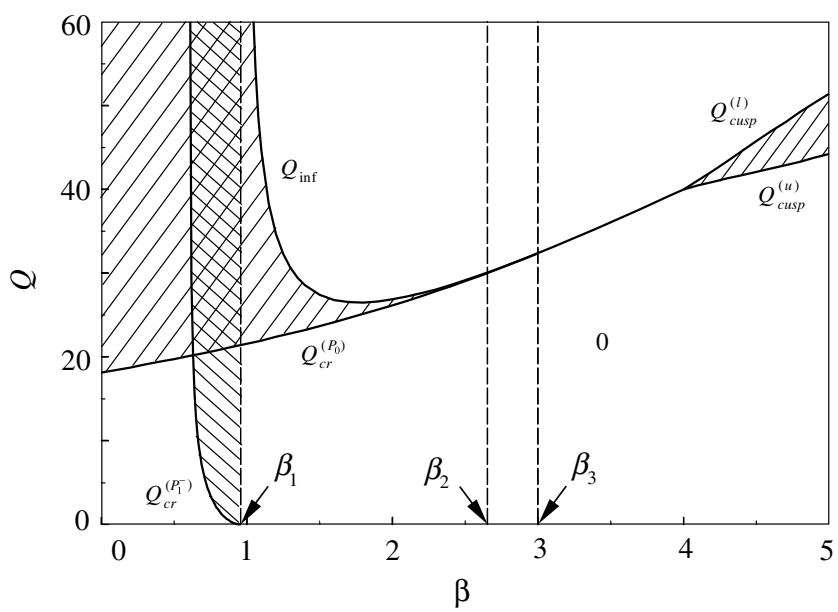

Fig. 8. Bifurcation set with $Q$ and $\beta$ as control parameters. The cross-hatched areas represent the parameter ranges in which static snap-through will occur.

ward at $e=1.60$. When the load is further increased to $Q_{\text {cusp }}^{(1)}=49.5$, the lower loop will degenerate to a cusp in the lower position. Therefore, static jumping of the arch occurs for the load between $Q_{\text {cusp }}^{(\mathrm{u})}$ and $Q_{\text {cusp }}^{(1)}$.

The equilibrium positions for various values of $\beta$ as discussed in Figs. 2-7 can be summarized in a bifurcation set diagram using $Q$ and $\beta$ as control parameters in Fig. 8 for an arch with $h=8$. The three vertical dashed lines represent $\beta_{1}, \beta_{2}$, and $\beta_{3}$. The cross-hatched areas represent the parameter ranges in which static snap through will occur. More specifically, the areas with positive slope lines represent the parameter range in which snap through will occur when the initial position of the $\operatorname{arch}$ is $P_{0}$. On the other hand, the area with negative slope lines represents the range in which snap through will occur when the initial position of the arch is $P_{1}^{-}$.

\section{Convergence test for dynamic response}

The response of the arch will be different when the point load travels with a nonzero speed. The response history can be calculated by integrating Eq. (9) numerically with the initial conditions (14). In the preceding section we have shown that the first four modes in the expansion are sufficient in static analysis. This does not guarantee that the same number of modes is sufficient in dynamic analysis. To test the convergence of the expansion, we calculate the response of an arch with $h=8, Q=23, \beta=0.5, c=2$ by using two, four, and eight modes in the expansion. In the numerical simulation we also add damping terms $\mu \dot{\alpha}_{j}$ in the equations of motion. Fig. 9(a) shows the calculated $\alpha_{1}$ for various numbers of modes used in the expansion. The damping parameter $\mu$ is chosen to be 0.001 . At the instant $\tau=1.57$ when the point force leaves the arch, as signified by a black dot in Fig. 9(a) the $\alpha_{1}$ calculated by using $N=4$ and 8 are almost indistinguishable. However, the arch will continue to vibrate after the point force leaves the arch until it settles to a steady state position. As shown in Fig. 9(a), the responses after the point load leaves the arch can be quite different for different number of modes used. For $N=2$, the calculated result shows that the arch will not snap, while for $N=4$ and $N=8$, the calculated results show that the arch will snap at time $\tau=51.89$ (not in the range of Fig. 9(a)) and 5.48, respectively. The convergence of $\alpha_{1}$ improves slightly when we further double the number of modes to 16 . However, $\alpha_{1}$ curves for $N=8$ and 16 still start to show significant discrepancy at time $\tau=8.64$. Our experience shows that the convergence deteriorates further when $\beta$ increases. 

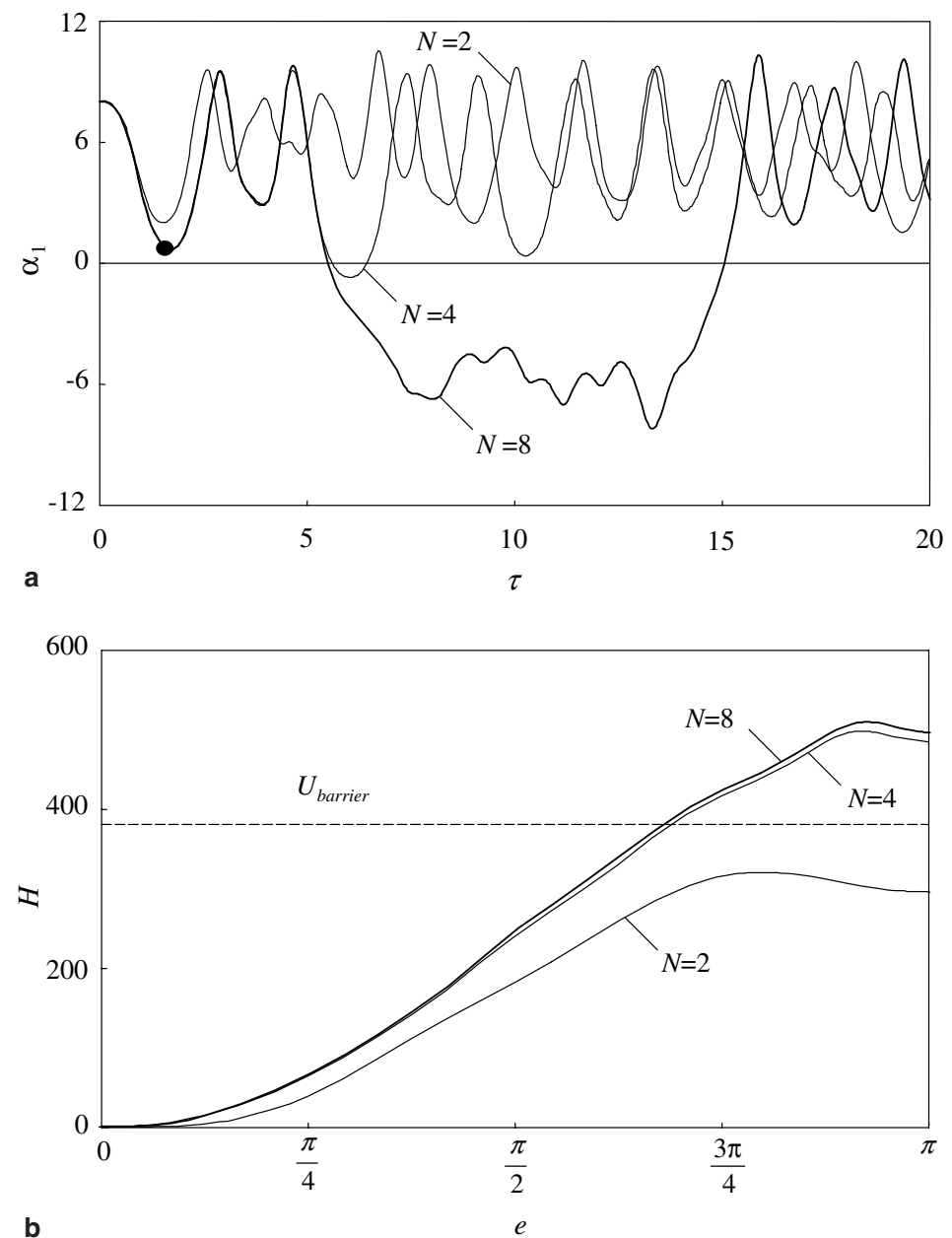

Fig. 9. Convergence test for dynamic response. (a) $\alpha_{1}$ does not converge well when $N=8$. (b) Total energy $H$ converges well when the first eight modes are used.

\section{Total energy and energy barrier}

Although it is difficult to predict the dynamic response $\alpha_{1}$ numerically after the point load leaves the arch, as demonstrated in Fig. 9(a), it does not jeopardize our goal of establishing a more conservative condition to guarantee the safe passage of the point force without snapping the arch. We first notice that the arch may snap either while the point load is still on the arch or after the point load leaves the arch. The snapping after the point load leaves the arch can occur only if the unloaded arch has two stable equilibrium positions, which is possible when $\beta<\beta_{2}$. Therefore, we can establish the following sufficient conditions to guarantee the safe passage of the load as follows. (1) The total energy $H$ gained by the arch at the instant when the point load reaches the other end is smaller than the critical energy barrier lying between the two distant stable equilibrium positions. (2) The coordinate $\alpha_{1}$ remains greater than zero while the point load is still on the arch. Condition (1) prevents the arch from snapping after the point load leaves the arch. Condition (2) guarantees that the arch does not snap while the point load is still on the arch. The total energy $H$ of the arch can be calculated as, 


$$
H=U+\sum_{n=1}^{\infty} \dot{\alpha}_{n}^{2}
$$

where $U$ is the strain energy of the arch and the elastic foundation,

$$
U=2 p^{2}+(1+\beta)\left(\alpha_{1}-h\right)^{2}+\sum_{n=2}^{\infty}\left[\left(n^{4}+\beta\right) \alpha_{n}^{2}\right] .
$$

The physical total energy $H^{*}$ and strain energy $U^{*}$ are related to $H$ and $U$ by

$$
H^{*}=\frac{\pi^{4} E I^{2} H}{4 A L^{3}}, \quad U^{*}=\frac{\pi^{4} E I^{2} U}{4 A L^{3}} .
$$

It can be shown that for an unloaded arch with $\beta<\frac{4}{11}$ and $h_{1}<h<h_{2}$, where

$$
h_{1}=4 \sqrt{1+\beta} \quad \text { and } \quad h_{2}=\frac{3|4-\beta|}{\sqrt{8-7 \beta}},
$$

the critical energy barrier $U_{\text {barrier }}$ is the strain energy $U$ of position $P_{1}^{+}$. On the other hand, for an arch with $h>h_{2}$ the critical energy barrier is the strain energy of position $P_{12}^{ \pm}$(Chen and Lin, 2004a). The two sufficient conditions can then be stated mathematically as

$$
\begin{aligned}
& H(e=\pi) \leqslant \operatorname{Min}\left[U\left(P_{1}^{+}\right), U\left(P_{12}^{ \pm}\right)\right], \\
& \alpha_{1}(0<e<\pi)>0 .
\end{aligned}
$$

Therefore, if we can predict the total energy $H$ at the instant when the point load leaves the arch accurately, we can estimate whether the arch is in danger of snapping either when the point load is still on the arch or after the point leaves the arch. Fig. 9(b) shows the total energy of the arch as in Fig. 9(a) when the point load moves across the arch. It is shown that the total energy calculated by using the first 4 and 8 modes in the expansion converge quite well. On the other hand, the first two modes are obviously insufficient. Further doubling the number of modes to 16 will not produce distinguishable difference in $H$ from the result calculated by using 8 modes. The critical energy barrier which corresponds to $U\left(P_{12}^{ \pm}\right)$is also plotted as a dashed horizontal line for comparison. In summary, while 8 modes are not sufficient in predicting the $\alpha_{1}$ after the load leaves the arch, it is sufficient in predicting whether the arch is in danger of snapping.

The fact that the total energy converges when 8 modes are used while the $\alpha_{1}$ response does not converge is not difficult to understand. We observe that the total energy and $\alpha_{1}$ at the instant when the point load leaves the arch are almost identical for $N=8$ and 16 . However, for $N=16$ the total energy can spill over to the sub-space $\alpha_{9}-\alpha_{16}$ due to nonlinear coupling of various modes, while for $N=8$ the total energy is confined to the space $\alpha_{1}-\alpha_{8}$. Therefore, as time goes by the $\alpha_{1}$ in these two cases will show significant difference while the total energy in these two cases remain almost unchanged.

\section{Dynamic snap through for a negative point load}

It is not difficult to envision the static and dynamic snap-through phenomena when the point load in Fig. 1 points downward, which is considered as positive in our model. On the other hand, when a negative point load moves onto the arch quasi-statically, it is obvious that the arch will not snap. However, it is not so obvious whether dynamic snap through can occur in this case. A high-speed cart moving on a roller coaster rail-track might be somewhat similar to this situation. If we consider the possibility that the arch may gain enough total energy to surpass the energy barrier, it becomes clear that the arch may snap after the load leaves the arch even when the point load is negative. In Fig. 10(a) we show the response $\alpha_{1}$ for an arch with $h=8, \beta=0.5$, and $Q=-36$. Two moving speeds $c=2.4$ and 2.8 are considered. The instants at which the 

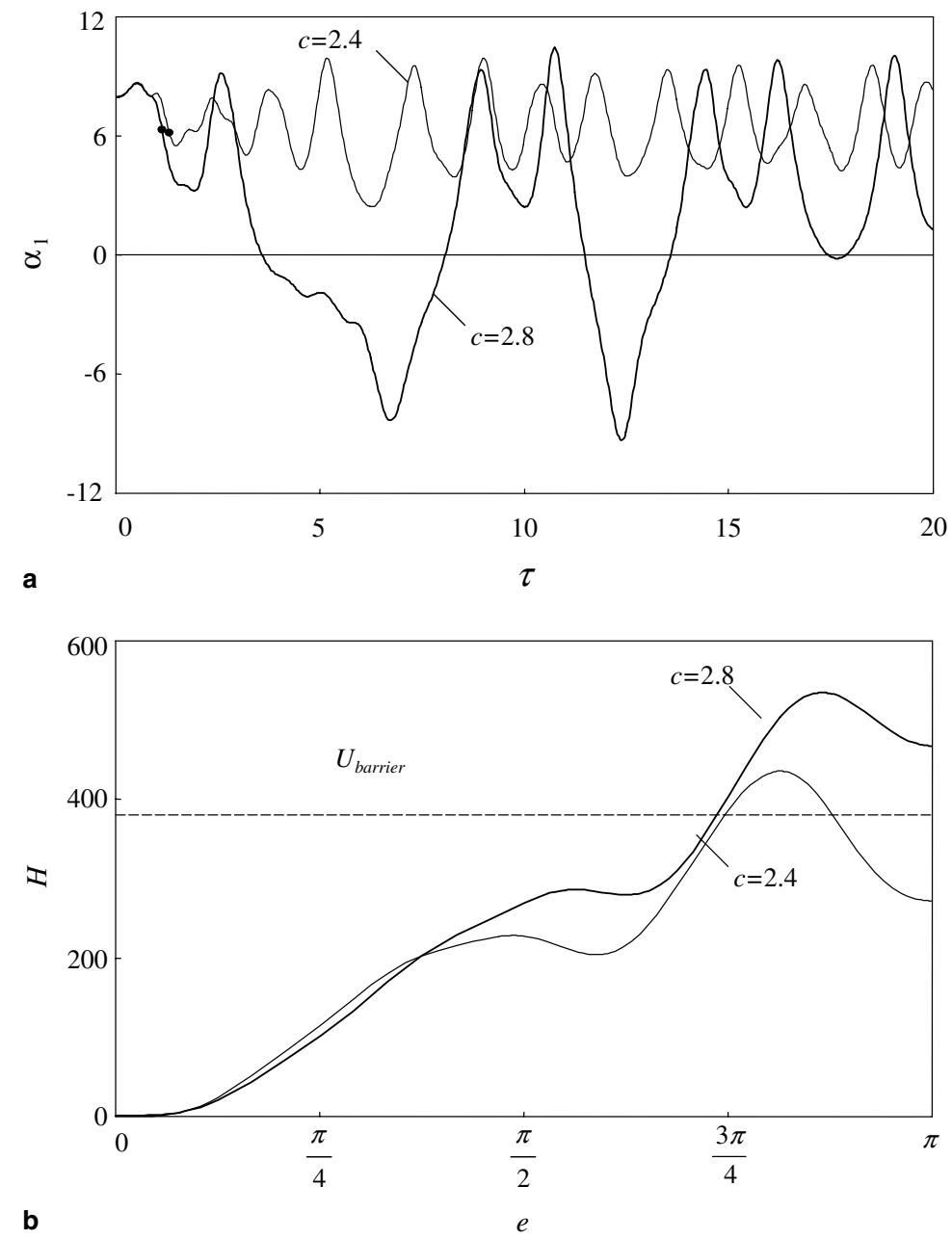

Fig. 10. (a) $\alpha_{1}(\tau)$ and (b) $H(e)$ for an arch under a negative point load $Q=-36, h=8$ and $\beta=0.5$.

point load leaves the arch are signified by black dots. It is observed that the $\alpha_{1}$ 's increase initially and were pulled back by the stiffness of the arch. At the instants when the loads leave the arch the arch is only flattened slightly but with high speed pointing downward. For the lower speed $c=2.4$, the arch will continue to oscillate but will not snap. On the other hand, for the higher speed $c=2.8$ the arch snaps back and forth several times in the time range of Fig. 10(a). The total energies gained by the arch and the elastic foundation with these two speeds as functions of load position are shown in Fig. 10(b). Obviously, at the instant when the point loads leave the arch, the total energy for the case $c=2.8$ exceeds the barrier while the total energy for $c=2.4$ does not.

\section{Boundary of dangerous speed zone}

In Fig. 11, we use the energy criterion to determine the boundary of the dangerous speed zone in the $Q-c$ plane for an arch with $h=8$ and various values of $\beta$. The arch is assumed to be in position $P_{0}$ before the 


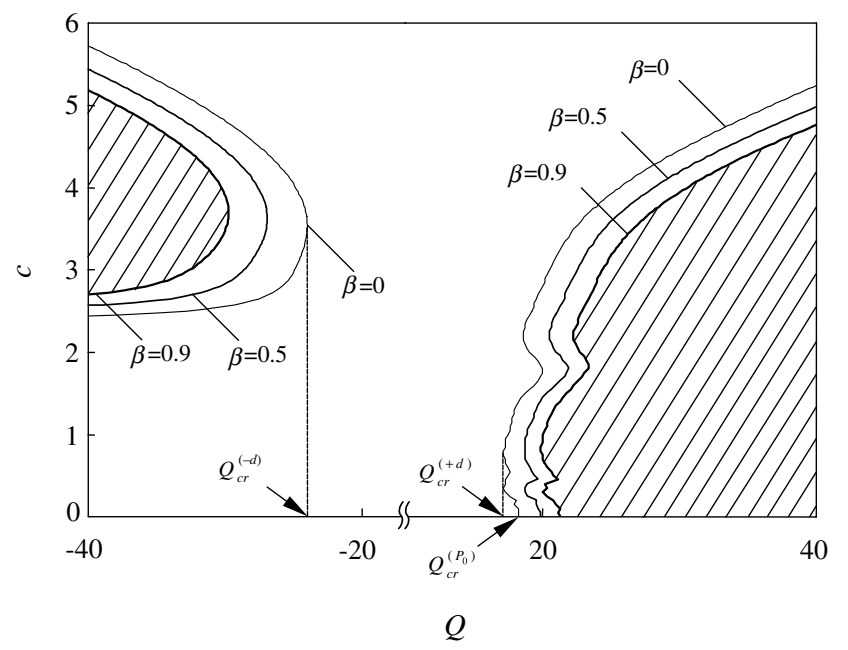

Fig. 11. Dangerous speed zone for an arch with $h=8$ and $\mu=0.001$ and various values of $\beta$.

load moves onto the arch. The point load can be positive or negative. We divide the parameter space in Fig. 11 into 100 by 100 points. For each of these parameter points we examine conditions (28) and (29) and locate the point at which the equal sign of condition (28) is satisfied. The damping coefficient $\mu$ is chosen to be 0.001 . The dangerous speed zone for $\beta=0.9$ is signified by the cross-hatched area. In other words, the arch runs the risk of snap-through if the parameter point $(Q, c)$ is in the shaded area. In the case when $c=0$, the critical loads $Q_{\mathrm{cr}}^{\left(P_{0}\right)}$ predicted from quasi-static analysis are 18.16, 19.77, and 21.21 for $\beta=0,0.5$, and 0.9 , respectively. On the other hand, no static snap-through will occur for negative point load. The positive dynamic critical load $Q_{\mathrm{cr}}^{+\mathrm{d}}$ is defined as the load below which the arch will not snap dynamically under positive load no matter what the moving speed is. The negative dynamic critical load $Q_{\mathrm{cr}}^{-\mathrm{d}}$ is defined similarly. The dynamic critical loads $Q_{\mathrm{cr}}^{+\mathrm{d}}$ and $Q_{\mathrm{cr}}^{-\mathrm{d}}$ for the case $\beta=0$ are signified in Fig. 11. In other words, the arch is safe from dynamic snap-through as long as the point load is in the range $Q_{\mathrm{cr}}^{-\mathrm{d}}<Q<Q_{\mathrm{cr}}^{+\mathrm{d}}$. Fig. 12 shows the

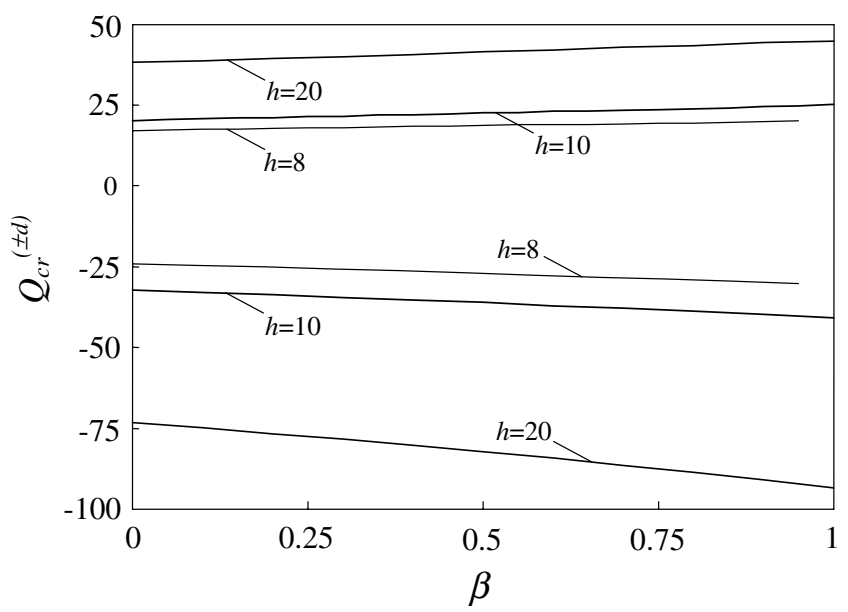

Fig. 12. Relation between the dynamic critical loads $Q_{\mathrm{cr}}^{( \pm \mathrm{d})}$ and $\beta$ for various values of $h$. 
relation between $Q_{\mathrm{cr}}^{ \pm \mathrm{d}}$ and $\beta$ for various values of $h$. Apparently, the safe range of $Q$ increases as the initial height of the arch and the spring constant of the foundation increase.

\section{Conclusions}

In this paper, we study the effects of elastic foundation on the static and dynamic snap-through of a shallow arch under a point load $Q$ traveling with a constant speed. Snap-through can occur when the arch is in either $P_{0}$ or $P_{1}^{-}$before the arch is loaded. For static analysis, the first four modes are sufficient in predicting the equilibrium positions. The evolution of the equilibrium positions for some typical values of elastic constant of the foundation is described in detail. On the other hand, the numerical simulation of the dynamic response does not converge well. In other words, it is almost impossible to predict the dynamic response of the arch numerically using only finite number of modes in the Fourier series. The convergence deteriorates as the spring constant of the foundation increases. Fortunately, the total energy of the arch converges quite well when only the first eight modes are used in the expansion. This observation allows us to predict whether the arch is in danger of dynamic snap-through, although we are unable to predict accurately at what time it will really occur. The sufficient condition which guarantees the safe passage of the point load without snapping the arch dynamically are based on the concept of comparing the total energy of the arch and the foundation at the instant when the point force leaves the arch and the critical energy barrier. With this method we can define a pair of dynamic critical loads $Q_{\mathrm{cr}}^{-\mathrm{d}}$ (negative) and $Q_{\mathrm{cr}}^{+\mathrm{d}}$ (positive), such that the arch is safe from snapping no matter what the moving speed is as long as the point load is in the range $Q_{\mathrm{cr}}^{-\mathrm{d}}<Q<Q_{\mathrm{cr}}^{+\mathrm{d}}$. The safe range of $Q$ increases as the initial height of the arch and the spring constant of the foundation increase.

\section{Acknowledgements}

The results presented here were obtained in the course of research supported by a grant from the National Science Council of the Republic of China.

\section{References}

Chen, J.-S., Liao, C.-Y., 2005. Experiment and analysis on the free dynamics of a shallow arch after an impact load at the end. ASME Journal of Applied Mechanics 72, 54-61.

Chen, J.-S., Lin, J.-S., 2004a. Effects of prescribed end motion on the dynamic stability of a shallow arch on an elastic foundation. ASCE Journal of Engineering Mechanics 130, 359-362.

Chen, J.-S., Lin, J.-S., 2004b. Dynamic snap-through of a shallow arch under a moving point load. ASME Journal of Vibration and Acoustics 126, 514-519.

Chen, J.-S., Lin, J.-S., 2005. Exact critical loads for a pinned half-sine arch under end couples. ASME Journal of Applied Mechanics $72,147-148$.

Donaldson, M.T., Plaut, R.H., 1983. Dynamic stability boundaries for a sinusoidal arch under pulse loads. AIAA Journal $21,469-471$.

Franciosi, V., Augusti, G., Sparacio, R., 1964. Collapse of arches under repeated loading. ASCE Journal of Structure Division 90, $165-201$

Fulton, R.E., Barton, F.W., 1971. Dynamic buckling of shallow arches. ASCE Journal of Engineering Mechanics Division 97, 865877.

Fung, Y.C., Kaplan, A., 1952. Buckling of low arches or curved beams of small curvature. NACA Technical Note 2840.

Gjelsvik, A., Bonder, S.R., 1962. The energy criterion and snap buckling of arches. ASCE Journal of Engineering Mechanics Division $88,87-134$.

Gregory Jr., W.E., Plaut, R.H., 1982. Dynamic stability boundaries for shallow arches. ASCE Journal of Engineering Mechanics Division 108, 1036-1050. 
Hetenyi, M., 1946. Beams on Elastic Foundation. The University of Michigan Press, Ann Arbor.

Hoff, N.J., Bruce, V.G., 1954. Dynamic analysis of the buckling of laterally loaded flat arches. Journal of Mathematics and Physics 32, 276-288.

Hsu, C.S., 1967. The effects of various parameters on the dynamic stability of a shallow arch. ASME Journal of Applied Mechanics 34 , 349-358.

Hsu, C.S., 1968. Stability of shallow arches against snap-through under timewise step loads. ASME Journal of Applied Mechanics 35, $31-39$.

Hsu, C.S., Kuo, C.T., Lee, S.S., 1968. On the final states of shallow arches on elastic foundations subjected to dynamical loads. ASME Journal of Applied Mechanics 35, 713-723.

Huang, N.N., Nachbar, W., 1968. Dynamic snap-through of imperfect viscoelastic shallow arches. ASME Journal of Applied Mechanics 35, 289-296.

Humphreys, J.S., 1966. On dynamic snap buckling of shallow arches. AIAA Journal 4, 878-886.

Johnson, E.R., 1980. The effect of damping on dynamic snap-through. ASME Journal of Applied Mechanics 47, $601-606$.

Johnson, E.R., Mclvor, I.K., 1978. The effect of spatial distribution on dynamic snap-through. ASME Journal of Applied Mechanics 45, 612-618.

Kerr, A.D., Dallis, W.A., 1985. Blowup of concrete pavements. ASCE Journal of Transportation Engineering 111, $33-53$.

Lee, H.N., Murphy, L.M., 1968. Inelastic buckling of shallow arches. ASCE Journal of Engineering Mechanics Division 94, $225-239$.

Lin, J.-S., Chen, J.-S., 2003. Dynamic snap-through of a laterally loaded arch under prescribed end motion. International Journal of Solids and Structures 40, 4769-4787.

Lo, D.L.C., Masur, E.F., 1976. Dynamic buckling of shallow arches. ASCE Journal of Engineering Mechanics Division 102, 901-917.

Lock, M.H., 1966. The snapping of a shallow sinusoidal arch under a step pressure load. AIAA Journal 4, $1249-1256$.

Onat, E.T., Shu, L.S., 1962. Finite deformation of a rigid perfectly plastic arch. ASME Journal of Applied Mechanics $29,549-553$.

Patricio, P., Adda-Bedia, M., Amar, M.B., 1998. An elastic problem: Instabilities of an elastic arch. Physica D 124, $285-295$.

Plaut, R.H., 1979. Influence of load position on the stability of shallow arches. Journal of Applied Mathematics and Physics (ZAMP) $30,548-552$.

Roorda, J., 1965. Stability of structures with small imperfections. ASCE Journal of Engineering Mechanics Division 91, 87-106.

Schreyer, H.L., Masur, E.F., 1966. Buckling of shallow arches. ASCE Journal of Engineering Mechanics Division 92, 1-19.

Simitses, G.J., 1973. Snapping of low pinned arches on an elastic foundation. ASME Journal of Applied Mechanics 40, 741-744.

Simitses, G.J., 1990. Dynamic Stability of Suddenly Loaded Structures. Springer-Verlag, New York.

Sundararajan, V., Kumani, D.S., 1972. Dynamic snap-buckling of shallow arches under inclined loads. AIAA Journal 10, $1090-1091$.

Timoshenko, S.P., 1935. Buckling of flat curved bars and slightly curved plates. ASME Journal of Applied Mechanics 2, 17-20.

Xu, J.-X., Huang, H., Zhang, P.-Z., Zhou, J.-Q., 2002. Dynamic stability of shallow arch with elastic supports-Application in the dynamic stability analysis of inner winding of transformer during short circuit. International Journal of Non-Linear Mechanics 37 , 909-920. 